\title{
Concentration dependence of solution shear viscosity and solute mass diffusivity in crystal growth from solutions
}

\author{
Alexander F. Izmailov and Allan S. Myerson \\ School of Chemical and Materials Science, Polytechnic University, Six MetroTech Center, Brooklyn, New York 11201
}

(Received 26 August 1994)

\begin{abstract}
The physical properties of a supersaturated binary solution such as its density $\rho$, shear viscosity $\eta$, and solute mass diffusivity $D$ are dependent on the solute concentration $c: \rho=\rho(c), \eta=\eta(c)$, and $D=D(c)$. The diffusion boundary layer equations related to crystal growth from solution are derived for the case of natural convection with a solution density, a shear viscosity, and a solute diffusivity that are all dependent on solute concentration. The solution of these equations has demonstrated the following. (a) At the vicinity of the saturation concentration $c_{s}$ the solution shear viscosity $\eta$ depends on $\rho$ as $\eta_{s}=\eta\left(\rho_{s}\right) \propto \rho^{1 / 2}\left(c_{s}\right)$. This theoretically derived result has been verified in experiments with several aqueous solutions of inorganic and organic salts. (b) The maximum solute mass transfer towards the growing crystal surface can be achieved for values of $c$ where the ratio of $d \ln [D(c) / d c]$ to $d \ln [\eta(c) / d c]$ is a maximum.
\end{abstract}

PACS number(s): 61.30. - v, 64.60.Cn, 64.60.My, 47.15.Cb

\section{INTRODUCTION}

The problem of crystal growth from supersaturated solutions is well known. In recent years there has been considerable interest in crystal growth under microgravity conditions. However, there is no appropriate formalism that accounts for both the hydrodynamic and the thermodynamic aspects of the problem. The development of such a formalism is of paramount importance in understanding how the static and the dynamic characteristics of a solution, such as its density, viscosity, and diffusivity, are related to each other at concentrations at saturation and above.

Current analytical approaches for the descriptions of crystal growth from supersaturated solutions (see [1-3] and references therein) neglect most of the significant features of the solution's metastable state. These include the nontrivial dependence of the solution density $\rho$, the shear $\eta$ and the bulk $\zeta$ viscosities, and the solute mass diffusivity $D$ on the solute concentration $c$. The usual practice [1-3] has been to assume that these physical characteristics are constants that are independent of solute concentration. However, recent studies [4-11] of supersaturated aqueous solutions of inorganic and organic salts have demonstrated that the solution density $\rho$, the shear viscosity $\eta$, and the solute mass diffusivity $D$ have a nontrivial dependence on the solute concentration $c$ : $\rho=\rho(c), \eta=\eta(c)$, and $D=D(c)$, which becomes more significant with deeper penetration into the metastable region. For example, the diffusivity $D(c)$ declines to zero at the spinodal line that separates metastable and unstable states. These facts have necessitated the development of an appropriate formalism to describe crystal growth from supersaturated solutions taking into account the dependence of these physical properties of supersaturated solutions on solute concentration.

\section{NATURAL CONVECTION EQUATIONS AND SOLUTION METASTABILITY}

Let us consider the situation where a supersaturated solution is mixed as a result of natural convection, which arises due to a depletion of solute concentration near the growing crystal surface. This depletion results in a change in solution density and leads to the appearance of concentration flows. However, because the solution has a finite viscosity and sticks to the crystal solid interface, an unmixed boundary layer is formed. Within this layer, adjacent to the growing crystal surface, the solution can be assumed to be stationary and the solute mass transfer is achieved only by means of ordinary diffusion. In this paper we assume that the crystal surface and the solution have the same temperature and thus there is no heat transfer.

Natural convection arises only when there is a change of solution density occurring in a gravitational field and only in those cases where the density gradient formed is perpendicular to a gravitational field or when the solution density increases from the bottom upward. The magnitude and the distribution of hydrodynamic and diffusional flows depend primarily on geometry and, in particular, on the shape and orientation of the growing crystal surface. Generally, on the basis of the Bjerkness theorem [12], one may expect that natural convection will occur in such a way that surfaces of equal solution density are oriented perpendicular to surfaces of equal pressure. In this paper we consider the simplest case where the growing crystal surface is a smooth vertical plate placed in a gravitational field. The case of horizontal orientation of the growing surface is considerably more complicated and will be studied later.

Solution metastability implies that its density, shear and bulk viscosities, and solute mass diffusivity are func- 
tions of solute concentration. Due to the crystal growth process the solute concentration is different far from the growing surface than at the crystal-liquid interface. It is usually assumed that an entire change of solute concentration occurs within the diffusion boundary layer (DBL) $\delta_{\text {diff }}$. At the growing crystal surface the solute concentration $c$ must be greater than or equal to the saturation concentration $c_{s}\left(c \geq c_{s}\right)$ at the system temperature and pressure. Outside the boundary layer the solute concentration is a constant $\left(c=c_{\infty}\right)$, although it fluctuates in time and space. Thus, within the boundary layer $\delta_{\text {diff }}$, for the case when the solution density $\rho(c)$, the shear $\eta(c)$ and the bulk $\zeta(c)$ viscosities, and the solute diffusivity $D(c)$ are weak functions of the solute concentration $c$ one can write the following Taylor expansions:

$$
\begin{aligned}
& \rho(c)=\rho\left(c_{\infty}\right)+\left.\frac{\partial \rho(c)}{\partial c}\right|_{c=c_{\infty}} \Delta c[1+O(1)], \\
& \eta(c)=\eta\left(c_{\infty}\right)+\left.\frac{\partial \eta(c)}{\partial c}\right|_{c=c_{\infty}} \Delta c[1+O(1)], \\
& D(c)=D\left(c_{\infty}\right)+\left.\frac{\partial D(c)}{\partial c}\right|_{c=c_{\infty}} \Delta c[1+O(1)],
\end{aligned}
$$

where $\Delta c=c-c_{\infty}$. The weakness concept for any infinitely times differentiable function $f(c)$ assumes that

$$
\left|\frac{d}{d c} \ln \frac{d^{n-1} f(c)}{d c^{n-1}}\right|_{c=c_{\infty}} \Delta c \ll n \text { where } n \geq 2 .
$$

In spite of the possible types of dependence of the function $f(c)$ on $c$, fulfillment of the weakness concept is always expected for the case of small supersaturations $|\Delta c|_{\max }=\left|c_{s}-c_{\infty}\right|$.

Let us take $x_{2}=0$ as the surface (010) of the growing crystal plate, directing the $x_{2}$ axis into a solution and the $x_{1}$ axis vertically upward. The lower ledge of the plate corresponds to the value $x_{1}=0$. As a supersaturated solution flows parallel to the crystal plate, the thickness of the boundary layer $\delta_{\text {diff }}$ increases with the distance $x_{1}$ from the lower ledge where flow meets the plate. Therefore, the thickness $\delta_{\text {diff }}$ of the DBL can be considered as an increasing function of $x_{1}: \delta_{\text {diff }}=\delta_{\text {diff }}\left(x_{1}\right)$. Thus the general Navier-Stokes equations describing the gravityinduced two-dimensional steady laminar motion of an incompressible supersaturated solution within $\left[x_{2} \leq \delta_{\text {diff }}\left(x_{1}\right)\right]$ and beyond $\left[x_{2}>\delta_{\text {diff }}\left(x_{1}\right)\right]$ the DBL acquire the form

$$
\begin{aligned}
& \sum_{j=1}^{2} \frac{\partial \Pi_{i j}}{\partial x_{j}}=\delta_{i l} g\left[\rho(c)-\rho\left(c_{\infty}\right)\right] \theta\left(\delta_{\mathrm{diff}}\left(x_{1}\right)-x_{2}\right), \\
& \frac{\partial v_{1}}{\partial x_{1}}+\frac{\partial v_{2}}{\partial x_{2}}=0
\end{aligned}
$$

where

$\Pi_{i j}=-p \delta_{i j}+\rho(c) v_{i} v_{j}+\eta(c)\left(\frac{\partial V_{i}}{\partial x_{j}}+\frac{\partial v_{j}}{\partial x_{i}}\right), i j=1,2$.

In the above expressions $\Pi_{i j}$ is the density tensor of the solution momentum flow, $p$ is the solution pressure, $v_{1}$ and $v_{2}$ are the solution velocity components, $g$ is the gravity acceleration, and $\theta\left(\delta_{\text {diff }}\left(x_{1}\right)-x_{2}\right)$ is the unit step function (Heaviside function) equal to 1 for $\delta_{\text {diff }}\left(x_{1}\right) \geq x_{2}$ and equal to 0 for $\delta_{\text {dif }}\left(x_{1}\right)<x_{2}$. Equation (4a) assumes that solution is isotropic, i.e., $\eta(c)$ is the scalar quantity. The continuity equation (4b) assumes that a supersaturated solution is an incompressible fluid. Utilizing the fact that the DBL thickness $\delta_{\text {diff }}\left(x_{1}\right)$ is very small compared to the length $L$ along a crystal plate, one can significantly simplify Eq. (4a). Thus, in the particular case where $\delta_{\text {diff }}\left(x_{1}\right) / L \ll 1$, the equation describing solution motion along the $x_{1}$ axis acquires the form

$$
\begin{aligned}
\rho(c) & {\left[\frac{\partial v_{1}}{\partial x_{1}} v_{1}+\frac{\partial v_{1}}{\partial x_{2}} v_{2}\right] } \\
\approx & -\frac{\partial}{\partial x_{2}}\left[\eta(c) \frac{\partial v_{1}}{\partial x_{2}}\right] \\
& +g\left[\rho(c)-\rho\left(c_{\infty}\right)\right] \theta\left(\delta_{\text {diff }}\left(x_{1}\right)-x_{2}\right) .
\end{aligned}
$$

This form implies the following assumptions imposed on solution motion: (a) viscosity exerts a significant influence only within a boundary layer, (b) flow velocity must become zero at the solution-crystal interface, (c) the solution retardation in a boundary layer is caused by viscous forces alone, and (d) the pressure change in a boundary layer is determined by its change outside and in the natural convection case is negligible since solution outside a boundary layer is almost stagnant. Substituting into Eq. (5) expansions (1) and (2), which account for the dependences of the solution density and the shear viscosity on solute concentration and keeping only the main terms proportional to $\Delta c$ and $\partial(\Delta c) / \partial x_{2}$ one can rewrite Eq. (5) as

$$
\begin{aligned}
\frac{\partial v_{1}}{\partial x_{1}} v_{1} & +\frac{\partial v_{1}}{\partial x_{2}} v_{2} \\
\approx & -v_{\infty} \frac{\partial^{2} v_{1}}{\partial x_{2}^{2}}+\omega_{\infty}\left[v_{\infty} \frac{\partial^{2} v_{1}}{\partial x_{2}^{2}}-g\right) \Delta c \theta\left(\delta_{\mathrm{diff}}\left(x_{1}\right)-x_{2}\right) \\
& -v_{\infty} \kappa_{\infty} \frac{\partial}{\partial x_{2}}\left[\frac{\partial v_{1}}{\partial x_{2}} \Delta c \theta\left(\delta_{\mathrm{diff}}\left(x_{1}\right)-x_{2}\right)\right]
\end{aligned}
$$

where

$$
\begin{aligned}
& v_{\infty}=\frac{\eta\left(c_{\infty}\right)}{\rho\left(c_{\infty}\right)}, \\
& \omega_{\infty}=\left.\frac{\partial \ln [\rho(c)]}{\partial c}\right|_{c=c_{\infty}}, \\
& \kappa_{\infty}=\left.\frac{\partial \ln [\eta(c)]}{\partial c}\right|_{c=c_{\infty}} .
\end{aligned}
$$

Boundary conditions for Eq. (6) have the form 


$$
\begin{aligned}
& \left.v_{1}\right|_{x_{2}=0}=\left.v_{2}\right|_{x_{2}=0}=0, \\
& \left.v_{1}\right|_{x_{2}=\infty}=\left.v_{2}\right|_{x_{2}=\infty}=0 .
\end{aligned}
$$

Condition (7a) reflects the fact that solution sticks to the crystal solid interface, whereas condition (7b) describes solution stagnancy at the infinite distance from the DBL edge. Thus Eqs. (4b) and (6), together with boundary conditions (7a) and (7b) describe the hydrodynamic aspects of natural convection corrected by accounting for solution metastability in the system supersaturated solution plus growing crystal surface.

\section{SOLUTION OF THE DERIVED EQUATIONS FOR THE CASE OF NATURAL CONVECTION IN SUPERSATURATED SOLUTIONS}

In order to simultaneously solve Eqs. (4b) and (6) let us introduce the stream function $\Psi\left(x_{1}, z\right)=\bigodot_{1} x_{1}^{3 / 4} f(z)$ and $z=e_{2} x_{1}^{-1 / 4} x_{2}$ defined by the relations $[12,13]$

$$
\begin{aligned}
& v_{1}=\frac{\partial \Psi\left(x_{1}, z\right)}{\partial x_{2}}=e_{1} e_{2} x_{1}^{1 / 2} f^{\prime}(z), \\
& v_{2}=-\frac{\partial \Psi\left(x_{1}, z\right)}{\partial x_{1}}=-\frac{e_{1}}{4} x_{1}^{-1 / 4}\left[3 f(z)-f^{\prime}(z)\right],
\end{aligned}
$$

where the dimension constants $e_{1}$ and $e_{2}$ and the function $f(z)$ are the quantities to be defined later. The introduction of the stream function $\Psi\left(x_{1}, z\right)$ allows us to rewrite Eq. (6) in the form

$$
\begin{aligned}
\frac{1}{2}\left[f^{\prime}(z)\right]^{2}-\frac{3}{4} f(z) f^{\prime \prime}(z)= & -v_{\infty} \frac{\varrho_{2}}{e_{1}} f^{\prime \prime \prime}(z)\left[1+\left(\kappa_{\infty}-\omega_{\infty}\right) \theta\left(\delta_{\mathrm{diff}}\left(x_{1}\right)-x_{2}\right) \Delta c\right] \\
& -\left[v_{\infty} \kappa_{\infty} \frac{\varrho_{2}}{\varrho_{1}} f^{\prime \prime}(z) \frac{\partial \Delta c}{\partial z}+\frac{g \omega_{\infty}}{\left(\varrho_{1} \bigodot_{2}\right)^{2}} \Delta c\right] \theta\left(\delta_{\mathrm{diff}}\left(x_{1}\right)-x_{2}\right)
\end{aligned}
$$

The equation obtained above can be satisfied only when the solute concentration $c$ is understood as a function of the variable z: $c=c(z)$. In addition, as described above, there exists the equality $c(z)=c_{\infty}$ for the case when $x_{2} \geq \delta_{\text {diff }}\left(x_{1}\right)$. This means that along the DBL edge the variable $z$ is constant: $z=z_{\infty}$. Therefore, after accomplishing straightforward but cumbersome calculations we come to the following important conclusions: (a) the DBL thickness $\delta_{\text {diff }}\left(x_{1}\right)$ is defined by the relation

$$
\delta_{\text {diff }}\left(x_{1}\right)=\frac{z_{\infty}}{\mathcal{C}_{2}} x_{1}^{1 / 4}
$$

and (b) in the vicinity of the DBL edge there exists the following expansion of $\Delta_{c}=\Delta c(z)$ :

$$
\Delta c(z)=\Gamma_{\infty}\left(z-z_{\infty}\right) \theta\left(z_{\infty}-z\right)+O\left(\left(z-z_{\infty}\right)^{2}\right), \quad \Gamma_{\infty}=\left.\frac{\partial c(z)}{\partial z}\right|_{z=z_{\infty}},
$$

where $\theta\left(z_{\infty}-z\right)$ is the Heaviside function [see definitions for expression (4a)]. Substituting now expansion (11b) into Eq. (6), we obtain the nonlinear differential equation for the function $f(z)$,

$$
\begin{aligned}
\frac{1}{2}\left[f^{\prime}(z)\right]^{2}-\frac{3}{4} f(z) f^{\prime \prime}(z)= & -v_{\infty} \frac{\varrho_{2}}{\varrho_{1}} f^{\prime \prime \prime}(z)\left[1+\left(\omega_{\infty}-\kappa_{\infty}\right) \Gamma_{\infty}\left(z-z_{\infty}\right) \theta\left(z_{\infty}-z\right)\right] \\
& +v_{\infty} \kappa_{\infty} \frac{\varrho_{2}}{\varrho_{1}} \Gamma_{\infty} f^{\prime \prime}(z) \theta\left(z_{\infty}-z\right)-\frac{g \omega_{\infty} \Gamma_{\infty}}{\left(\bigodot_{1} \varrho_{2}\right)^{2}}\left(z-z_{\infty}\right) \theta\left(z_{\infty}-z\right) .
\end{aligned}
$$

Let us look for a solution of this equation in the form

$$
f(z)=f_{w}(z) \theta\left(z_{\infty}-z\right)+f_{b}(z) \theta\left(z-z_{\infty}\right),
$$

where the functions $f_{w}(z)$ and $f_{b}(z)$ give solutions of Eq. (12) within and beyond the DBL. Equations for the function $f_{w}(z)$ and $f_{b}(z)$ have the forms

$$
\begin{aligned}
& \frac{1}{2}\left[f_{w}^{\prime}(z)\right]^{2}-\frac{3}{4} f_{w}(z) f_{w}^{\prime \prime}(z) \\
& =-v_{\infty} \frac{\bigodot_{2}}{\bigodot_{1}} f_{w}^{\prime \prime \prime}(z)\left[1+\left(\omega_{\infty}-\kappa_{\infty}\right) \Gamma_{\infty}\left(z-z_{\infty}\right)\right] \\
& \quad+v_{\infty} \kappa_{\infty} \frac{\bigodot_{2}}{\bigodot_{1}} \Gamma_{\infty} f_{w}^{\prime \prime}(z)-\frac{g \omega_{\infty} \Gamma_{\infty}}{\left(\bigodot_{1} \bigodot_{2}\right)^{2}}\left(z-z_{\infty}\right),
\end{aligned}
$$

$$
\frac{1}{2}\left[f_{b}^{\prime}(z)\right]^{2}-\frac{3}{4} f_{b}(z) f_{b}^{\prime \prime}(z)=-v_{\infty} \frac{\bigodot_{2}}{\bigodot_{1}} f_{b}^{\prime \prime \prime}(z) .
$$

Boundary conditions for these equations given in terms of the functions $f_{w}(z)$ and $f_{b}(z)$ can be derived from relations $(7)-(9)$ in the form

$$
\begin{aligned}
& \left.f_{w}(z)\right|_{z=0}=\left.f_{w}^{\prime}(z)\right|_{z=0}=0, \\
& \left.f_{b}(z)\right|_{z=\infty}=\left.f_{b}^{\prime}(z)\right|_{z=\infty}=0 .
\end{aligned}
$$

One of the possible solutions of Eq. (14a) subjected to the boundary condition (15a), can be found as the series expansion 


$$
f_{w}(z)=\sum_{n=0}^{\infty} \frac{f_{n}}{n !} z^{n},
$$

where

$$
\begin{aligned}
& f_{0}= f_{1}=f_{2}=0 \\
& f_{3}= \frac{g \omega_{\infty} \Gamma_{\infty} z_{\infty}}{v_{\infty} e_{1} e_{2}^{3}\left[1+\left(\kappa_{\infty}-\omega_{\infty}\right) \Gamma_{\infty} z_{\infty}\right]}, \\
& f_{4}= \frac{\kappa_{\infty} \Gamma_{\infty} z_{\infty}-1}{1+\left(\kappa_{\infty}-\omega_{\infty}\right) \Gamma_{\infty} z_{\infty}^{2}} f_{3} \\
& f_{5}= \frac{3 \kappa_{\infty}-2 \omega_{\infty}}{1+\left(\kappa_{\infty}-\omega_{\infty}\right) \Gamma_{\infty} z_{\infty}} f_{4} \\
& f_{0}= \frac{4 \kappa_{\infty}-3 \omega_{\infty}}{1+\left(\kappa_{\infty}-\omega_{\infty}\right) \Gamma_{\infty} z_{\infty}} f_{5}, \\
& f_{n+1}= \frac{(n-1) \kappa_{\infty}-(n-2) \omega_{\infty}}{1+\left(\kappa_{\infty}-\omega_{\infty}\right) \Gamma_{\infty} z_{\infty}} f_{n} \\
&-\sum_{m=3}^{n-3} \frac{(n-2) !}{m !(n-m) !}\left[5 m(n-m)-\frac{3}{2} n(n-1)\right] \\
& \quad \times f_{m} f_{n-m},
\end{aligned}
$$

where $n \geq 6$. This solution can be considerably simplified if one assumes that the solution constant $z_{\infty}$ can be defined as $z_{\infty}=1 /\left(\Gamma_{\infty} \kappa_{\infty}\right)$. Such an assumption leads to the vanishing of all expansion coefficients $f_{n}(n \geq 4)$ leaving only one nonzero term in series (16) corresponding to $n=3$,

$$
f_{w}(z)=\frac{1}{6} f_{3} z^{3}, \quad f_{3}=\frac{g \omega_{\infty}}{v_{\infty} e_{1} e_{2}^{3}\left(2 \kappa_{\infty}-\omega_{\infty}\right)}
$$

One of the possible solutions of Eq. (14b) subjected to the boundary condition $(15 b)$ is

$$
f_{b}(z)=-12 v_{\infty} \frac{e_{2}}{e_{1}} \frac{1}{z}
$$

Both solutions $f_{w}(z)$ and $f_{b}(z)$ obtained correspond to connective flows within and beyond the DBL and should coincide along this layer edge $f_{w}\left(z_{\infty}\right)=f_{b}\left(z_{\infty}\right)$. This condition provides a way to determine the constant $e_{2}$ in the form

$$
e_{2}=\frac{1}{\Gamma_{\infty} \kappa_{\infty}}\left[\frac{g \omega_{\infty}}{72 v_{\infty}^{2}\left(\omega_{\infty}-2 \kappa_{\infty}\right)}\right]^{1 / 4} .
$$

It is noteworthy that further agreement (patching) of the solutions $f_{w}(z)$ and $f_{b}(z)$ is impossible since their first derivative and all consequent derivatives are not equal along the DBL edge. Therefore, one may conclude that the problem general solution $f(z)$, given by relation (13), has discontinuity points along the DBL edge. To explain this conclusion let us find velocity components $v_{1}$ and $v_{2}$ within and beyond the DBL. For this purpose results (17a) and (17b) for the functions $f_{w}(z)$ and $f_{b}(z)$ have to be substituted into the corresponding expressions (8) and (9) for the velocity components:

$$
\begin{aligned}
& v_{1}=\frac{g \omega_{\infty}}{2 v_{\infty}\left(2 \kappa_{\infty}-\omega_{\infty}\right)} x_{2}^{2}, v_{2}=0 \text { (within), } \\
& v_{1}=12 v_{\infty} \frac{x_{1}}{x_{2}^{2}}, \quad v_{2}=12 v_{\infty} \frac{1}{x_{2}} \text { (beyond). }
\end{aligned}
$$

It follows from the expressions obtained for the velocity components $v_{1}$ and $v_{2}$ that within the DBL the tangent velocity component $v_{1}$ is infinitely times greater than its normal component $v_{2}$, whereas beyond the DBL these components become of the same order. This corresponds to the following well-understood phenomenon of the DBL separation: (a) within the DBL the solution motion occurs along the crystal surface without side deviations and (b) along the DBL edge the tangent and normal velocity components become of the same order and the DBL separation takes place. Thus the DBL edge can be identified with the discontinuity line in solution motion $[13,14]$.

\section{THE DBL THICKNESS AND RELATIONSHIP BETWEEN SOLUTION SHEAR VISCOSITY AND DENSITY AT SATURATION POINT}

Substituting relation (8) into expression (11a) for the DBL thickness $\delta_{\text {diff }}\left(x_{1}\right)$ we obtain the result

$$
\delta_{\text {diff }}\left(x_{1}\right)=\left[\frac{72 v_{\infty}^{2}\left(2 \kappa_{\infty}-\omega_{\infty}\right) x_{1}}{|g| \omega_{\infty}}\right]^{1 / 4} .
$$

In the expression above we have taken into account that

TABLE I. Dependence of solution density $\left(\mathrm{g} / \mathrm{cm}^{3}\right)$ on solute concentration (mass fraction). The saturation point is taken at $25^{\circ} \mathrm{C}$ and normal pressure.

\begin{tabular}{lccccc}
\hline \hline $\begin{array}{l}\text { Aqueous } \\
\text { solution }\end{array}$ & $\begin{array}{c}\varrho \text { of } \\
\eta_{\text {sample }}\left(c_{\infty}\right)\end{array}$ & $a_{0}$ & $a_{1}$ & $\begin{array}{c}\text { Saturation point } \\
\text { at } 25^{\circ} \mathrm{C} \\
\text { and normal pressure } \\
\text { (in mass fraction) }\end{array}$ & $\begin{array}{c}\text { Intersection point } \\
\text { between } \eta\left(c_{\infty}\right) \\
\text { and } \eta_{\text {sample }}\left(c_{\infty}\right) \\
\text { (in mass fraction) }\end{array}$ \\
\hline $\mathrm{KCl}$ & 0.904 & 1.013 & 0.571 & 0.264 & 0.268 \\
$\mathrm{ADP}$ & 1.739 & 0.998 & 0.564 & 0.283 & 0.253 \\
$\mathrm{KDP}$ & 1.294 & 0.996 & 0.744 & 0.200 & 0.195 \\
TGS & 1.437 & 1.005 & 0.367 & 0.231 & 0.215 \\
glycine & 1.329 & 1.000 & 0.343 & 0.198 & 0.183 \\
\hline \hline
\end{tabular}


the $x_{1}$ axis and the gravity acceleration $g$ are oppositely directed. Thus, by means of the result (19), we have derived how the hydrodynamics of natural convection are related to thermodynamic metastability of supersaturated solutions. In expression (19) metastability effects are taken into account through the dependence of the solution density $\rho\left(c_{\infty}\right)$ and the shear viscosity $\eta\left(c_{\infty}\right)$ on the bulk solute concentration $c_{\infty}$.

An analysis of expression (19) obtained for the DBL thickness $\delta_{\text {diff }}\left(x_{1}\right)$ allows the following conclusions. First, we have obtained the well-known result that $\delta_{\text {diff }}\left(x_{1}\right)$ grows as $\left(x_{1} /|g|\right)^{1 / 4}$ (see [12-14] and references therein). Second, we have derived how the DBL thickness $\delta_{\text {diff }}\left(x_{1}\right)$ depends on the bulk solute concentration $c_{\infty}$ via such solution static and dynamic characteristics as its density $\rho\left(c_{\infty}\right)$ and viscosity $\eta\left(c_{\infty}\right)$. This dependence provides an opportunity to relate to each other the supersaturated solution static and dynamic characteristics. In particular, expression (19) allows a relationship between the density $\rho\left(c_{s}\right)$ and the viscosity $\eta\left(c_{s}\right)$ of supersaturated
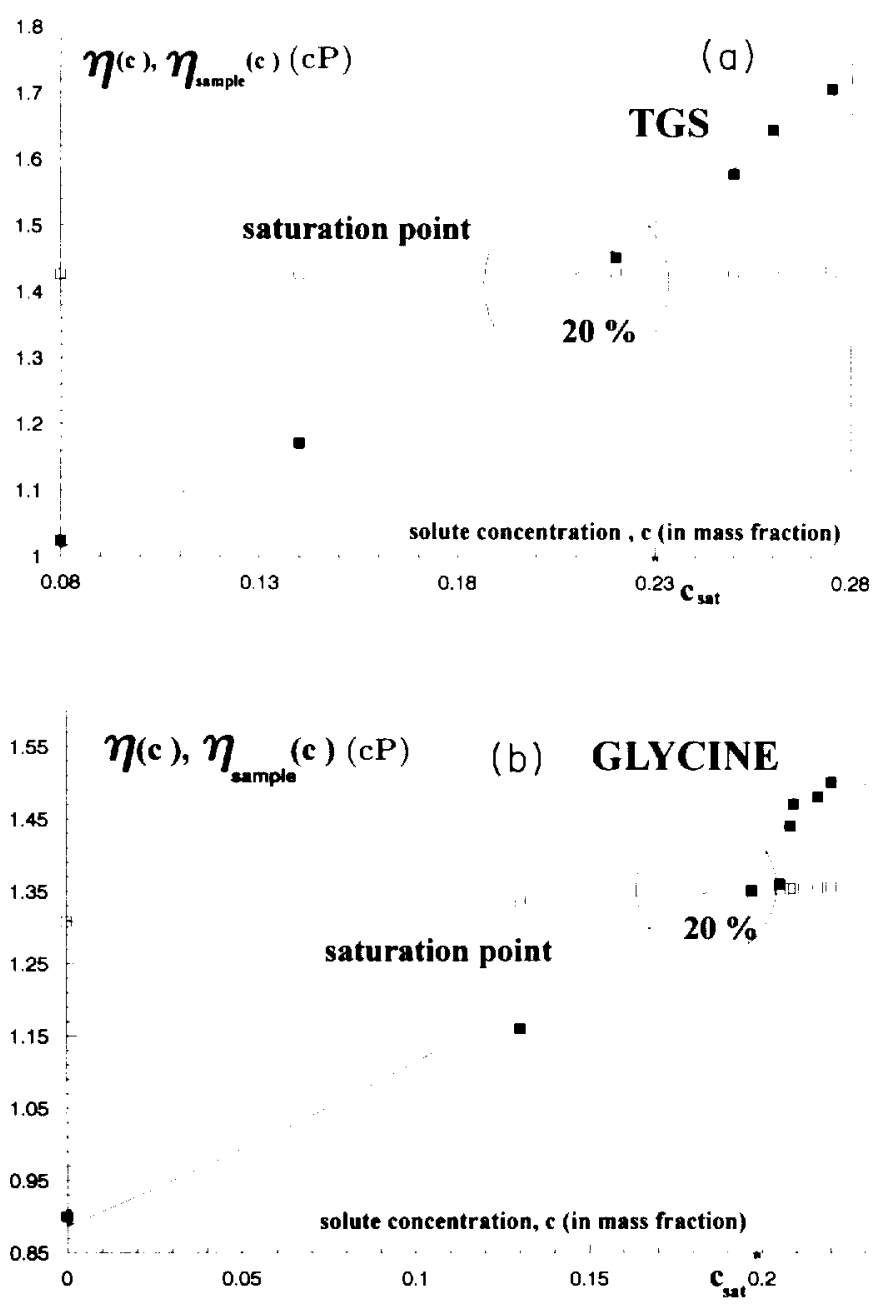

FIG. 1. Dependence of the bulk solution viscosity $\eta\left(c_{\infty}\right)$ (cP) on the bulk solute concentration $c_{\infty}$ (mass fraction) for the TGS and glycine aqueous solutions. Solid lines correspond to experimental data, whereas short-dashed lines correspond to interpolation by means for the sample function $\eta_{\text {sample }}\left(c_{\infty}\right)$. solutions. It is apparent that a boundary layer should vanish at the saturation point since at this point solution and the growing crystal surface are in thermodynamic equilibrium. As it follows from the result (19) obtained for $\delta_{\text {diff }}\left(x_{1}\right)$, such a situation is possible only when the following equality is satisfied:

$$
\left.\omega_{\infty}\right|_{c_{\infty}=c_{s}}=\left.2 \kappa_{\infty}\right|_{c_{\infty}}=c_{s} .
$$

An analysis of Eq. (20) gives that at saturation point the solution density $\rho\left(c_{s}\right)$ and viscosity $\eta\left(c_{s}\right)$ should be related as

$$
\eta\left(c_{s}\right)=e \rho^{1 / 2}\left(c_{s}\right)
$$

This result has been experimentally verified with inorganic and organic aqueous solutions such as $\mathrm{NaCl}, \mathrm{KCl}$, urea, ADP $\left(\mathrm{NH}_{4} \mathrm{H}_{2} \mathrm{PO}_{4}\right), \quad \mathrm{KDP} \quad\left(\mathrm{KH}_{2} \mathrm{PO}_{4}\right), \quad$ TGS $\left[\left(\mathrm{C}_{3} \mathrm{H}_{5} \mathrm{NO}_{2}\right)_{3} \mathrm{H}_{2} \mathrm{SO}_{4}\right]$, and glycine [4-11] taken at $25^{\circ} \mathrm{C}$ and normal pressure. For all these solutions it was found that the dependence of their bulk densities $\rho\left(c_{\infty}\right)$ on the bulk solute concentration $c_{\infty}$ was linear: $\rho\left(c_{\infty}\right)=a_{0}+a_{1} c_{\infty}$ (Table I gives coefficients $a_{0}$ and $a_{1}$ for different solutions). The error of such a linear interpolation of the experimental density data was always within $0.01 \%$. The experimentally obtained dependences of the solution shear viscosity $\eta\left(c_{\infty}\right)$ on the bulk solute concentration $c_{\infty}$ for the TGS and glycine aqueous solution are presented in Figs. 1(a) and 1(b) (the experimental error of the viscosity measurements is within $15 \%$ ). In these figures the solid lines correspond to the viscosity experimental data versus solute concentration whereas short-dashed lines represent the sample function $\eta_{\text {sample }}\left(c_{\infty}\right)=e \rho^{1 / 2}\left(c_{\infty}\right)$. It follows from the straightforward comparison between the viscosity experimental data line and the sample function line that their intersection approximately corresponds (the error in correspondence is within $15 \%$ ) to the saturation concentration $c_{s}$ at the given temperature and pressure for every tested solution (see Table 1). Therefore, the analytically derived conclusion that at the vicinity of the saturation point there is the specific relationship, given by expression (21), between the solution viscosity and the density is experimentally confirmed with an accuracy of $85 \%$.

\section{SOLUTE FLOW TOWARDS THE GROWING CRYSTAL SURFACE}

To define a complete system of equations describing isothermal solute diffusion in the natural convection case one has to supplement the general Navier-Stokes and continuity equations (4a) and ( $4 b$ ) by the corresponding solute diffusion equation. For the particular case where a supersaturated solution can be considered as an incompressible fluid and in the stationary limit, the twodimensional equation for the convective solute diffusion acquires the form

$$
\frac{\partial c}{\partial x_{1}} v_{1}+\frac{\partial c}{\partial x_{2}} v_{2}=\frac{\partial}{\partial x_{1}}\left[D(c) \frac{\partial c}{\partial x_{1}}\right]+\frac{\partial}{\partial x_{2}}\left[D(c) \frac{\partial c}{\partial x_{2}}\right] \text {. }
$$


It is assumed in this equation that for the saturated and the supersaturated solutions the solute mass diffusivity $D(c)$ is dependent on the solute concentration $c$. The evident analytical form of this dependence is not established yet. However, numerous experimental investigations [4-11] have demonstrated that $D(c)$ is a nontrivial and strong function of the solute concentration $c$. In order to solve Eq. (22) within the DBL, it is assumed that there exists an expansion (3) for the diffusivity $D(c)$. The following utilization of the fact that the DBL thickness $\delta_{\text {diff }}\left(x_{1}\right)$ is very small compared to the characteristic length $L$ of a crystal plate allows one to considerably simplify Eq. (22):

$$
\begin{aligned}
\frac{\partial c}{\partial x_{1}} v_{1} & +\frac{\partial c}{\partial x_{2}} v_{2} \\
& =D\left(c_{\infty}\right)\left[\frac{\partial^{2} c}{\partial x_{2}^{2}}+\gamma_{\infty}\left[\frac{\partial c}{\partial x_{2}}\right]^{2} \theta\left(\delta_{\text {diff }}\left(x_{1}\right)-x_{2}\right)\right],
\end{aligned}
$$

where

$$
\gamma_{\infty}=\left.\frac{\partial \ln [D(c)]}{\partial c}\right|_{c=c_{\infty}} .
$$

It is noteworthy that $\gamma_{\infty}<0$ since in the supersaturated region the solute mass diffusivity $D\left(c_{\infty}\right)$ is a decreasing function of the solute concentration $c_{\infty}$ : the diffusivity $D\left(c_{\infty}\right)$ declines to zero when $c_{\infty}$ is approaching spinodal concentration at the given temperature and pressure.

To solve Eq. (23) let us introduce the new couple $\left(x_{1}, z\right)$ of independent variables, replacing $\left(x_{1}, x_{2}\right)$ by $\left(x_{1}, z\right)$ :

$$
\frac{\partial}{\partial x_{1}}=\frac{\partial}{\partial x_{1}}-\frac{z}{4 x_{1}} \frac{\partial}{\partial z}, \frac{\partial}{\partial x_{2}}=e_{2} x_{1}^{-1 / 4} \frac{\partial}{\partial z} .
$$

The following replacement of the velocity components $v_{1}$ and $v_{2}$ by their expressions given by relations (8) and (9) allows one to rewrite Eq. (23) in terms of the new couple of independent variables:

$$
\begin{aligned}
& f^{\prime}(z) \frac{\partial c}{\partial x_{1}}-\frac{3 f(z)}{4 x_{1}} \frac{\partial c}{\partial z} \\
& =\frac{\mathcal{C}_{2}}{\mathcal{C}_{1}} \frac{D\left(c_{\infty}\right)}{x_{1}}\left[\frac{\partial^{2} c}{\partial z^{2}}+\gamma_{\infty}\left(\frac{\partial c}{\partial z}\right)^{2} \theta\left(z_{\infty}-z\right)\right],
\end{aligned}
$$

where the function $f(z)$ within and beyond the DBL is given by relations (17a) and (17b), respectively. Taking into account the conclusion, obtained in the analysis of Eq. (10), that within the DBL the solute concentration $c$ is a function of the only variable $z, c=c(z)$, provides the following simplification of Eq. (24):

$$
-\frac{3}{4} f(z) \frac{\partial c}{\partial z}=\frac{e_{2}}{e_{1}} D\left(c_{\infty}\right)\left\lfloor\frac{\partial^{2} c}{\partial z^{2}}+\gamma_{\infty}\left[\left.\frac{\partial c}{\partial z}\right|^{2} \theta\left(z_{\infty}-z\right)\right]\right. \text {. }
$$

In terms of the variable $z$, boundary conditions for this equation can be reduced to the form

$$
\left.c(z)\right|_{z=0}=c(0),\left.\quad \frac{\partial c}{\partial z}\right|_{z=0}=\beta_{0}\left[c(0)-c_{s}\right],
$$

where $c(0)$ is the solute concentration on the growing crystal surface $(z=0)$. It is natural to assume that in the crystal growing regime there exists the following double inequality: $c_{s} \leq c(0) \leq c_{\infty}$. The constant $\beta_{0}=\beta(c(0)) \geq 0$ is the $c(0)$-dependent coefficient that characterizes the rate of solute exchange between the crystal surface and the solution. The second boundary condition (26) describes such a situation where the solute mass flow towards a crystal surface is positive if $c(0) \geq c_{s}$.

The solution of Eq. (25) subjected to boundary conditions (26) is straightforward:

$$
c(z)=c_{w}(z) \theta\left(z_{\infty}-z\right)+c_{b}(z) \theta\left(z-z_{\infty}\right) .
$$

In this expression $c_{w}(z)$ and $c_{b}(z)$ are the solutions for the solute concentration profiles within and beyond the DBL, respectively,

$$
\begin{aligned}
& c_{w}(z)=c(0)+\frac{1}{\gamma_{\infty}} \ln \left[1+\gamma_{\infty} z_{\infty} \beta_{\infty}\left[c(0)-c_{s}\right]\right. \\
& \left.\quad \times \int_{0}^{z / 2} d x e^{(9 / 4) S c x^{4}}\right], \\
& c_{b}(z)=c_{\infty},
\end{aligned}
$$

where $\mathrm{Sc}=v_{\infty} / D\left(c_{\infty}\right)$ is the Schmidt number. The analysis of the solution $c(z)$ for $z=0$ and $z_{\infty}$ provides a possibility to determine the coefficient $\beta_{0}$ as the following monotonically decreasing (without local extremum) function of $c(0)$ :

$\beta_{0}=\frac{1}{\gamma_{\infty} z_{\infty}} \frac{e^{\left.\gamma_{\infty} \mid c_{\infty}-c(0)\right\}}-1}{c(0)-c_{s}}\left|\int_{0}^{1} d x e^{(9 / 4) \mathbf{S c x} x^{4}}\right|^{-1}$.

Substituting this expression for the coefficient $\beta_{0}$ back into relation (27) for the solute concentration $c_{w}(z)$ we obtain

$$
\begin{aligned}
& c_{w}(z)=c(0)+\frac{1}{\gamma_{\infty}} \ln \mid 1+\left(e^{\gamma_{\infty}\left[c_{\infty}-c(0)\right]}-1\right) \\
& \times \frac{\int_{0}^{z / z} d x e^{(9 / 4) \mathrm{Sc} x^{4}}}{\int_{0}^{1} d x e^{(9 / 4) \mathrm{Sc} x^{4}}}
\end{aligned}
$$

Differentiating this expression with respect to $z$ and having in mind the relationship (11b), one can derive the following condition imposed on the problem charcteristics:

$$
F(1)=1-e^{\gamma_{\infty}\left[c(0)-c_{\infty}\right]},
$$

where

$$
F\left(\frac{z}{z_{\infty}}\right)=\frac{\gamma_{\infty}}{\kappa_{\infty}} e^{-(9 / 4) \mathrm{Sc}} \int_{0}^{z / z} d x e^{(9 / 4) \mathrm{Sc} x^{4}} .
$$

It is noteworthy that $F\left(z / z_{\infty}\right)<0$ since $\gamma_{\infty}<0$ in the supersaturated region. Utilization of condition (30) allows one to rewrite the result (29) for the solute concentration 
$c_{w}(z)$ profile as the following function of the variable $z$ :

$$
c_{w}(z)=c(0)+\frac{1}{\gamma_{\infty}} \ln \left(1+\frac{F\left(\frac{z}{z_{\infty}}\right)}{1-F(1)}\right) .
$$

Taking into account that $z=z_{\infty} x_{2} / \delta_{\text {diff }}\left(x_{1}\right)$, one can represent expression ( $31 \mathrm{a}$ ) for $c_{w}(z)$ in terms of the initial coordinate variables $x_{1}$ and $x_{2}$,

$$
c_{w}(z)=c(0)+\frac{1}{\gamma_{\infty}} \ln \left(1+\frac{F\left(\frac{x_{2}}{\delta_{\mathrm{diff}}\left(x_{1}\right)}\right)}{1-F(1)}\right) .
$$

In the crystal growth problem it is essential to know the solute diffusional flux $j_{\text {diff }}\left(x_{1}, x_{2}\right)$ directed towards the growing crystal surface. This flux is defined as

$$
j_{\mathrm{diff}}(z)=D\left(c_{w}\right) \frac{\partial c_{w}(z)}{\partial z}=e_{2}^{-1} x_{1}^{1 / 4} D\left(c_{w}\right) \frac{\partial c_{w}(z)}{\partial x_{2}} .
$$

Let us find separately the flux $j_{\text {diff }}(z)$ on the DBL edge $\left[x_{2}=\delta_{\text {diff }}\left(x_{1}\right)\right.$ or $\left.z=z_{\infty}\right]$ and on the crystal surface $\left(x_{2}=0\right.$ or $\left.z=0\right)$ :

$j_{\text {diff }}\left(x_{1}, \delta_{\text {diff }}\left(x_{1}\right)\right)=D\left(c_{\infty}\right) \Gamma_{\infty}$,

$j_{\text {diff }}\left(x_{1}, 0\right)=j_{\text {diff }}\left(x_{1}, \delta_{\text {diff }}\left(x_{1}\right)\right) \frac{D(c(0))}{D\left(c_{\infty}\right)} \frac{e^{-(9 / 4) \mathrm{Sc}}}{1-F(1)}$,

where $D(c(0))$ is the solute mass diffusivity on crystal surface and $e_{2}$ is given by expression (18). Having in mind that within the DBL the solute diffusivity $D(c)$ is a weak function of the solute concentration $c$ [an assumption already used in expansion (3)], one may represent $D(c(0))$ in the form:

$$
D(c(0))=D\left(c_{\infty}\right)\left(1-\frac{\gamma_{\infty}}{\kappa_{\infty}}\right) .
$$

Therefore, the ratio $\lambda\left(\gamma_{\infty}, \kappa_{\infty}, \mathrm{Sc}\right)=j_{\mathrm{diff}}\left(x_{1}, 0\right) /$ $j_{\text {diff }}\left[x_{1}, \delta_{\text {diff }}\left(x_{1}\right)\right]$, which characterizes the efficiency of the solute mass transfer towards the growing crystal surface, is given by the expression

$\lambda\left(\gamma_{\infty}, \kappa_{\infty}, \mathrm{Sc}\right)=\lambda(\epsilon, \mathrm{Sc})=\frac{(1+\epsilon) e^{-(9 / 4) \mathrm{Sc}}}{1+\epsilon e^{-(9 / 4) \mathrm{Sc}} \int_{0}^{1} d \xi e^{(9 / 4) \mathrm{Sc} \xi^{4}}}$,

where $\epsilon=\left|\gamma_{\infty}\right| / \kappa_{\infty}$. The analysis of this expression gives that the ratio $\lambda(\epsilon, \mathrm{Sc})$ is the monotonic function of the both variables $\epsilon$ and Sc. However, $\lambda(\epsilon, \mathrm{Sc})$ is the increasing function of the variable $\epsilon(0 \leq \epsilon \leq \infty)$, whereas it is the decreasing function of the variable Sc $(0 \leq \mathrm{Sc} \leq \infty)$. For the given Schmidt number Sc the ratio $\lambda(\epsilon, \mathrm{Sc})$ acquires a minimum value when $\epsilon=0$ and a maximum when $\epsilon=\infty$ :

$$
\begin{aligned}
& \min \left[\left.\lambda(x, \mathrm{Sc})\right|_{\mathrm{Sc}=\text { const }}\right]=\lambda(0, \mathrm{Sc})=e^{-(9 / 4) \mathrm{Sc}}, \\
& \begin{aligned}
\max \left[\left.\lambda(x, \mathrm{Sc})\right|_{\mathrm{Sc}=\text { const }}\right] \\
=\lambda(\infty, \mathrm{Sc})=\left(\int_{0}^{1} d \xi e^{-(9 / 4) \mathrm{Sc} \xi^{4}}\right)^{-1} .
\end{aligned}
\end{aligned}
$$

Therefore, the most favorable regime for the solute mass transfer corresponds to such solute concentration regions where the ratio of $d \ln [D(c)] / d c$ to $d \ln [\eta(c)] / d c$ acquires a maximum value. Expression (34b) for the ratio $\lambda(\infty, \mathrm{Sc})$ can be considerably simplified if one makes use of the Watson lemma [15] in the calculation of the integral involved. It is straightforward to demonstrate that in the limiting case where $\mathrm{Sc} \gg \frac{4}{9}$ there exists an approximation

$$
\int_{0}^{1} d \xi e^{(9 / 4) \mathrm{Sc} \xi^{4}} \approx \frac{1}{9 \mathrm{Sc}} e^{(9 / 4) \mathrm{Sc}} .
$$

Substitution of this result back into relation (34b) provides the following simple expression for the ratio $\lambda(\infty, \mathrm{Sc})$ :

$$
\lambda(\infty, \mathrm{Sc})=9 \mathrm{Sc} e^{-(9 / 4) \mathrm{Sc}},
$$

where $\mathrm{Sc} \gg \frac{4}{9}$.

It is the usual situation when the solute mass diffusivity $D(c)$ is a stronger function of the solute concentration $c$ than the solution shear viscosity $\eta(c)$. Therefore, the case of $\epsilon=0$ corresponds to a complete disregard of the $c$ dependence in diffusivity as well as in viscosity. On the contrary, the case of $\epsilon=\infty$ describes such concentration regions where any change of solute concentration leads to the much faster changing of the $c$-dependent diffusivity than of the $c$-dependent viscosity. It is noteworthy that accounting for the $c$ dependence of the solute mass diffusivity $D(c)$ and the solution shear viscosity $\eta(c)$ leads to an increase of the ratio $\lambda(\epsilon, \mathrm{Sc})$ estimations $\lambda(0, \mathrm{Sc})<\lambda(\infty, \mathrm{Sc})$.

\section{CONCLUSIONS}

It follows from the analysis presented above that (a) in the vicinity of the saturation concentration $c_{s}$ there exists the relationship between the solution shear viscosity and density, $\eta\left(\rho_{s}\right) \propto \rho^{1 / 2}\left(c_{s}\right)$; (b) the most favorable regimes for the solute mass transfer towards the growing crystal surface can be achieved when $\epsilon \gg 1$; and (c) the estimations of solute mass flow towards the growing crystal surface performed within the approaches disregarding the $c$ dependence of $D(c)$ and $\eta(c)$ lead to its underestimation.

It is well known that under microgravity conditions one may expect a significant improvement in crystal growth since the DBL thickness increases with the decrease of the gravity acceleration constant $g$ [see expression (19)]. However, as it follows from expression (19), it is not necessarily the case. For example, at low supersaturations the ratio $\left(2 \kappa_{\infty} / \omega_{\infty}-1\right) / g$ can still be small even at microgravity conditions. This prevents the formation of the appropriate boundary layer needed for improvement of the crystal growth process. Thus, to achieve such an improvement of crystal growth one has to obtain supersaturations so that $2 \kappa_{\infty} / \omega \gg 1$.

\section{ACKNOWLEDGMENTS}

The authors gratefully acknowledge the support of the National Science Foundation (Grant No. CTS 9020233) and NASA (Grant No. NAG8-960). 
[1] J. C. Brice, The Growth of Crystals from Liquids, Selected Topics in Solid State Physics Vol. XII (North-Holland, Amsterdam, 1973).

[2] F. Rosenberger, Fundamentals of Crystal Growth, edited by Editor(s), Springer Series in Solid-State Sciences Vol. V (Springer-Verlag, Berlin, 1979).

[3] A. A. Chernov, in Modern Crystallography III. Crystal Growth, edited by Editor(s), Springer Series in Solid-State Sciences Vol. 36 (Springer-Verlag, Berlin, 1984).

[4] L. Sorell and A. S. Myerson, AIChE J. 28, 772 (1984).

[5] Y. C. Chang and A. S. Myerson, AIChE J. 30, 820 (1984).

[6] Y. C. Chang and A. S. Myerson, AIChE J. 31, 890 (1985).

[7] Y. C. Chang and A. S. Myerson, AIChE J. 32, 1567 (1986).

[8] P. Y. Lo and A. S. Myerson, J. Cryst. Growth 110, 20
(1990).

[9] R. M. Ginde and A. S. Myerson, AIChE Symp. Ser. 87, 124 (1991).

[10] R. M. Ginde and A. S. Myerson, J. Cryst. Growth 116, 41 (1992).

[11] M. Bohenek (unpublished).

[12] H. Schlichting, Boundary Layer Theory (McGraw-Hill, New York, 1960).

[13] L. D. Landau and E. M. Lifshitz, Fluid Mechanics (Pergamon, London, 1976), Vol. VI.

[14] Y. Jaluria, Natural Convection. Heat and Mass Transfer (Pergamon, Oxford, 1980).

[15] L. Sirovich, Techniques of Asymptotic Analysis, Applied Mathematical Sciences Vol. 2 (Springer, New York, 1971). 